\title{
Effect of Cognitive-Behavioral Therapy Focused on Self- esteem on Fear of Childbirth: A Counseling Approach in Prenatal Care and a Randomized Clinical Trial
}

\author{
Maryam Poorjandaghi ${ }^{1}$, Katayon Vakilian ${ }^{2 *}$, Mahboobeh Khorsandi ${ }^{3}$, Mansour Abdi $^{4}$
}

\begin{abstract}
Objectives: This study aimed to evaluate the effect of cognitive-behavioral therapy focused on self-esteem (CBT-S) on fear of childbirth in nulliparous women.

Materials and Methods: In this randomized clinical trial, 24 nulliparous women willing to cesarean section delivery referred to Milad hospital, Tehran, Iran, between July to August 2015 were enrolled with a convenient sampling method. Participants were assigned into two CBT-S and control groups (24 in each group) using randomized blocks of 4 . Seven sessions were performed for the CBT-S group, but the control group received routine prenatal classes. FOC was assessed using Harman's childbirth attitudes questionnaire. The Rosenberg self-esteem scale was used to assess self-esteem at the beginning of the study. Two weeks after the intervention, both groups filled out the FOC and self-esteem questionnaire.

Results: The mean score of fear at the end of the study in the CBT-S and control group was significantly different $(P=0.001)$. Also, selfesteem scores increased after intervention in the CBT-S counseling group $(4.77 \pm 4.07 \mathrm{vs}-1.79 \pm 2.26 ; P=0.001)$.

Conclusions: This study confirms the importance of the CBT-S approach focusing on self-esteem in reducing FOC.

Keywords: Natural childbirth, Education, Self-efficacy, Antenatal care, Fear of childbirth.
\end{abstract}

\section{Introduction}

It seems that $7 \%$ of primiparous women experience severe childbirth fear (1). In many countries, including Iran, childbirth fear has increased the demand for cesarean delivery by women $(2,3)$. The cesarean section occurs to many fears and anxiety; such as fear of unbearable pain, the anxiety of death, fear of unknown events, failure of a vaginal birth (natural childbirth), fear of the death of the fetus or newborn, and lack of support by medical personnel (4). Social-ecological model shows that our feeling and behaviors are affected by an environmental, individual, interpersonal, organizational, community and culture (5). Fear of childbirth (FOC) feels that affected by individual, Medical, social and cultural factors such as maternal age, history of prenatal and delivery complications, low education, low socioeconomic level, psychiatric problems, low self-esteem personality, and lack of social support (6)

The failure to recognize mothers' fear by midwives and their lack of understanding of their concerns about natural childbirth can lead to some pregnancy period problems such as fatigue, heartburn, and sleep disorders; and some delivery problems including preterm labor, prolonged labor, dystocia; and some postpartum problems such as the weak maternal-infant bonding $(7,8)$.
Studies have shown that non-realistic fears of natural delivery are accompanied by cognitive distortions such as a sense of invaluableness and lack of ability to have natural childbirth. They are potent predictors in FOC $(9,10)$. A sense of self-worth or self-esteem is the belief that individuals hold about their values and importance. Self-esteem consists of self-respect and self-efficacy. Selfefficacy is one's ability to make appropriate decisions and do things well. Self-respect having assurance in oneself, that is, to give ourselves the right to a happy life. Having a high level of self-esteem improves mental health and is a protective factor against mental illnesses such as anxiety, depression, phobias, and lack of happiness $(2,10)$. It seems that the use of counseling methods has been effective in overcoming childbirth fear. In this regard, various counseling methods have been tried, including psycho-education intervention $(11,12)$, Yoga (13), and the cognitive behavioral therapy (CBT) (14). Research illustrates that traditional training for childbirth cannot effectively address issues such as reducing stress during pregnancy related to maternal mental health (15). A study examined CBT in pregnancy and revealed that these approaches are safe, applicable, and effective in managing anxiety disorders in pregnancy (14). CBT has a theoretical 


\section{Key Messages}

- FOC is an essential experience in childbirth that can be controlled by psycho-education methods such as CBT-S.

framework that provides a way for understanding and treating psychological problems. The cognitivebehavioral approach focuses on how problematic beliefs and behaviors play a role in developing psychological problems and, much more importantly, in maintaining these problems over time $(16,17)$.

The aim of this study was to investigate the effect of CBT-S on FOC in nulliparous women under prenatal care in Iran.

\section{Materials and Methods}

Study Design and Participants

In this randomized clinical trial, 24 nulliparous women willing to cesarean section delivery referred to Milad hospital, Tehran, Iran, between July to August 2015 were enrolled with a convenient sampling method.

The inclusion criteria were as follows age between 1830 years old, high school diploma as a minimum level of education, singleton pregnancy, nulliparity, low-risk pregnancy (lack of chronic and acute diseases, having a normal body mass index (BMI) $\left(18.6-25.0 \mathrm{~kg} / \mathrm{m}^{2}\right)$, no history of psychiatric and psychological illnesses, no panic disorder of childbirth, lack of history of infertility, lack of any family conflicts, the score of zero and less than zero on the Rosenberg's self-esteem scale, and median score of 28 was considered as the cut-off point for FOC.

The exclusion criteria included lack of cooperation, withdrawal from the research for any reason, lack of constant attendance in the counseling sessions (more than two absences in a class session), and the termination of pregnancy due to fetal death.

\section{Sample Size}

The sample size of 24 for each group was calculated according to $\alpha=0.5, \beta=0.2$, standard deviation of 27.9 and the mean difference $\left(\chi 1-\chi^{2}=8\right)$ base on Khorsandi and colleagues' study (18), and used the following formula:

$$
\frac{\left(s 1^{2}+s 2^{2}\right)\left(Z_{1-\frac{\alpha}{2}+z 2_{1-\beta}}\right)^{2}}{(x 1-x 2)^{2}}
$$

$$
(x 1-x 2)^{2}
$$

\section{Randomization}

Participants were assigned into two CBT-S and control groups (24 in each group) using randomized blocks of 4 . Six variants were written on six separate sheets of paper folded and put into a plastic bag. The women asked to pick one of the sheets. For example, the combination of $A B A B$ led the first person to the CBT-S counseling group (A) and the second person was assigned into the control group, the third and fourth subjects were allocated to the counseling groups similarly; this continued until 48 subjects were allocated to each group.

Interventions

A counseling program was conducted by a student in midwifery counseling who completed a CBT course, and the supervisor supervised some sessions. The program was provided for 90 minutes once a week with a total of 8 sessions. At the end of each counseling session, the CBT-S counseling group performed the relaxation technique (Table 1). In the control group, mothers participated in preparation for childbirth classes during eight sessions. Before and two weeks after the program was finished, the Harman's childbirth attitude questionnaire and selfesteem questionnaire were completed by the intervention and control groups.

\section{Data Collection}

A third-part questionnaire was used for data collection. The first part collected demographic information. Harman's childbirth attitudes questionnaire was employed to assess FOC in the second part. This questionnaire revised by Lowe includes 14 questions rated on a four-point Likert's scale (never, seldom, sometimes, and often) with a total score range from 14 to 56 , in which a higher score indicates more fear 2. According to similar international studies, a median score of 28 was considered the cut-off point for FOC (2). The validity of the FOC questionnaire was confirmed based on the Khorsandi and co-workers study in Iran, and its reliability was determined using Cronbach's alpha ( $\alpha=0.85)$ (19).

Rosenberg's self-esteem questionnaire evaluates the participants' self-esteem level in the third part. This questionnaire is a four-point Likert scale consisting of ten statements, measuring overall life satisfaction and feeling good about oneself. The agree response to each statement 1 to 5 receives $(+1)$; the disagree response to each statement 1 to 5 receives $(-1)$; they agree response to each statement 6 to 10 receives $(+1)$, and the disagree response to each statement 6 to 10 receives $(-1)$. The scores range between $(-10)$ and $(+10)$. The scores higher than zero present higher levels of self-esteem, and the scores less than zero indicate lower self-esteem (20). In the present study, pregnant nulliparous women who received a score of zero or less were included in the study.

\section{Outcomes}

Two weeks after CBT-S sessions in the intervention group and in the end of the study in the control group, FOC and self-esteem of pregnant women were assessed by Harman's childbirth attitudes and Rosenberg's self-esteem questionnaire, respectively

Data Analysis

Data were analyzed by using the Statistical Package 
Table 1. The Content of Sessions in Self-esteem Integrated Cognitive Behavior Approach

- Introducing the trainer to the group members and saying welcome to the participants,

- Making the participants familiar with each other

- Explaining the aim and rules of sessions

Session - Determining the general duties of the group members

1 - Asking the participants questions about their awareness of the fear of childbirth and how to deal with it

- Making participants familiar with the overall concept of the fear of childbirth; its causes and its impact on a person's life, pregnancy, and childbirth; and the importance of the childbirth fear prevention and control

- Relaxation practice

- Checking the participants' mood

- Making participants familiar with the cognitive-behavioral approach:

- Explaining the interactive nature of the three systems of thought, behavior, and emotion

- Training the ABC mode- (A) Activator event, (B) Beliefs or thoughts, (C) Its emotional consequence

- Empowering the participants in receiving superficial, introductory thoughts that are between the event and emotional reaction, and writing them in the form of three columns, and practicing them in the class as role-playing

- Relaxation practice

- Checking the participants' mood

- Reviewing the previous sessions

- Familiarizing the members with the cognitive distortions and empowering them to identify these intellectual errors (training the cognitive errors including the catastrophizing, black or white thinking, prediction, mind reading, emotional reasoning, overgeneralization, labeling, mental filtering, disregarding good things, do's and don'ts, magnification, personalization, etc.).

The way of coping with the cognitive errors (using a self-assessment model) was provided to the participants, which included: a) Write down your feelings;

Session $\quad$ b) Write down your behaviors and reactions (how your behavior changes when you feel uncomfortable);

$3 \quad$ c) Write down the factor (activating event) causing this feeling;

d) Explain what the event meant to you (writing beliefs, thoughts, and attitudes);

e) Identify your cognitive errors;

f) Find a solution for any of the destructive ideas, beliefs, and attitudes;

g) Write your own opinion about the impact of the new thought on your feeling;

h) Assign some tasks for yourself.

- Relaxation practice

- Checking the participants' mood

- Reminding and emphasizing (summation) the contents of previous sessions with the cooperation of the participants

- Talking about the questions raised about the contents of the previous session;

- Checking the given task;

Session - Differentiating healthy negative emotions from unhealthy negative emotions;

- Using proper words to express the negative feelings, for instance, instead of saying "I'm angry", to tell "I'm upset";

- Understanding that unhealthy negative emotions are due to cognitive errors that can be transformed into healthy negative emotions (a practice in the classroom);

- Relaxation practice

- Asking about the mood of each mother (checking the mood)

- Reviewing and emphasizing (samation) the contents of previous sessions with the cooperation of the participants;

- Talking about the questions raised on the contents of the previous session

- Checking the given task;

Session - Familiarity with the concept of self-esteem and its importance and effect on the childbirth fear;

5 - Identifying the characteristics of individuals with high self-esteem and their recognition in the subjects;

- Familiarity with barriers to self-esteem caused by cognitive distortions;

- Teaching effective coping strategies with barriers to self-esteem;

- Presentation of a task (think of yourself in the third person view and write down your characteristics, abilities, weaknesses, and accomplishments);

- Relaxation practice

- Asking about the mood of each mother (checking the mood)

- Reviewing and emphasizing (samation) the contents and statements of previous sessions with the cooperation of the participants;

- Talking about the questions raised on the contents of previous sessions;

- Checking the given task:

Session - Definition of self-acceptance (presented as a way to raise self-esteem in the class);

6 - Training the principles of self-esteem;

- Action to accept yourself (talking to yourself as your best friend); for instance, imagine being your best friend, talk to yourself, accept and understand your mistakes as your best friend's mistakes, then write them and describe how you evaluate yourself and your problems considering the situation.

- Relaxation practice

- Asking about the mood of each mother (checking the mood)

- Reviewing and emphasizing (samation) the contents and statements of previous sessions with the cooperation of the participants;

- Talking about the questions raised on the contents of previous sessions;

- Checking the given task;

Session - An overview of the contents of the previous session

7 - Reviewing your plans for changing your thoughts and behaviors in the future;

- Displaying short videos of childbirth experiences and a CD of natural childbirth to watch at home with the spouse

- Practicing meditation at home;

- Encouraging yourself to continue the exercises;

- Asking about the mood of each mother (checking the mood)

- Reviewing and emphasizing (summation) the contents of previous sessions with the cooperation of the participants;

Session - Talking about the questions raised on the contents of the previous session;

- Providing written feedback from members to each other and the therapist;

- Relaxation practice

- Saying goodbye and determining the date of the post-test. 
for the Social Sciences software (SPSS, version 16.0 for Windows; SPSS Inc., Chicago, IL), and the level of 0.05 was considered statistically significant. The normal distribution of quantitative data was measured using the Kolmogorov-Smirnov test. Independent Student's $t$ test, chi-square, Mann-Whitney $\mathrm{U}$, and Wilcoxon tests were used to determine the statistical significance in the mean of self-esteem and fear of childbirth between two groups.

\section{Results}

Out of 100 pregnant women, 48 (24 in each group) women expressed their willingness to attend to research. Due to the exclusion of two participants for uncompleted sessions, data from 46 participants were analyzed (Figure 1). Demographic characteristics such as marital status and gestational age in the two groups were compared in Table 2.

The mean of FOC and self-esteem at the beginning study in the intervention and control groups have been presented in table 3 , indicating that there is no significant difference between the two groups in FOC score (39.59 vs. 41.17; $P=0.376)$ and self-esteem score at baseline $(-2.09$ vs. $-1.88 ; P=0.678)$. The other findings bring out in Table 3. The results indicated that the levels of fear and selfesteem significantly increment in the SICB group.

\section{Discussion}

This study aimed to examine the effect of CBT-S counseling in FOC. According to our findings, FOC in pregnant women could reduce by raising self-esteem in cognitive-behavioral approach. In one study the effect
CBT. The study used cognitive therapy techniques for FOC based on a six-session group therapy in 3 weeks in Turkish women was assessed. The results of Turkish study showed that birth-related concerns (including FOC and pain) were reduced using cognitive therapy, and the length of labor was shorter in the intervention group (14). The other study also revealed the positive effects of psychoeducative group therapy in nulliparous women with fewer depressive symptoms and FOC (21).

In the present study, cognitive technique, which generally means identifying negative automatic thoughts and replacing them with positive thoughts through obtaining knowledge and assessing the situation using cognitive exercises, could reduce FOC. Mothers were familiarized with their irrational and rational beliefs and their schemas. They found that their childbirth fear was not actually due to unpleasant events but resulted from their beliefs. So, they could discover the source of their childbirth fear and concerns by recognizing and classifying their beliefs in rational and irrational beliefs. The mothers were able to manage negative events through training in cognitive distortions, dealing with them, designing and implementing behavioral experiences that all these were new steps to gain social support.

The use of educational videos, seeing the women who had a successful and pleasant childbirth experience, and the labor support provided by the midwives created a sense of value and respect in the participants, increased their self-esteem, and improved their attitude towards childbirth fear. The mothers were taught how to overcome their scary thoughts when experiencing negative emotions

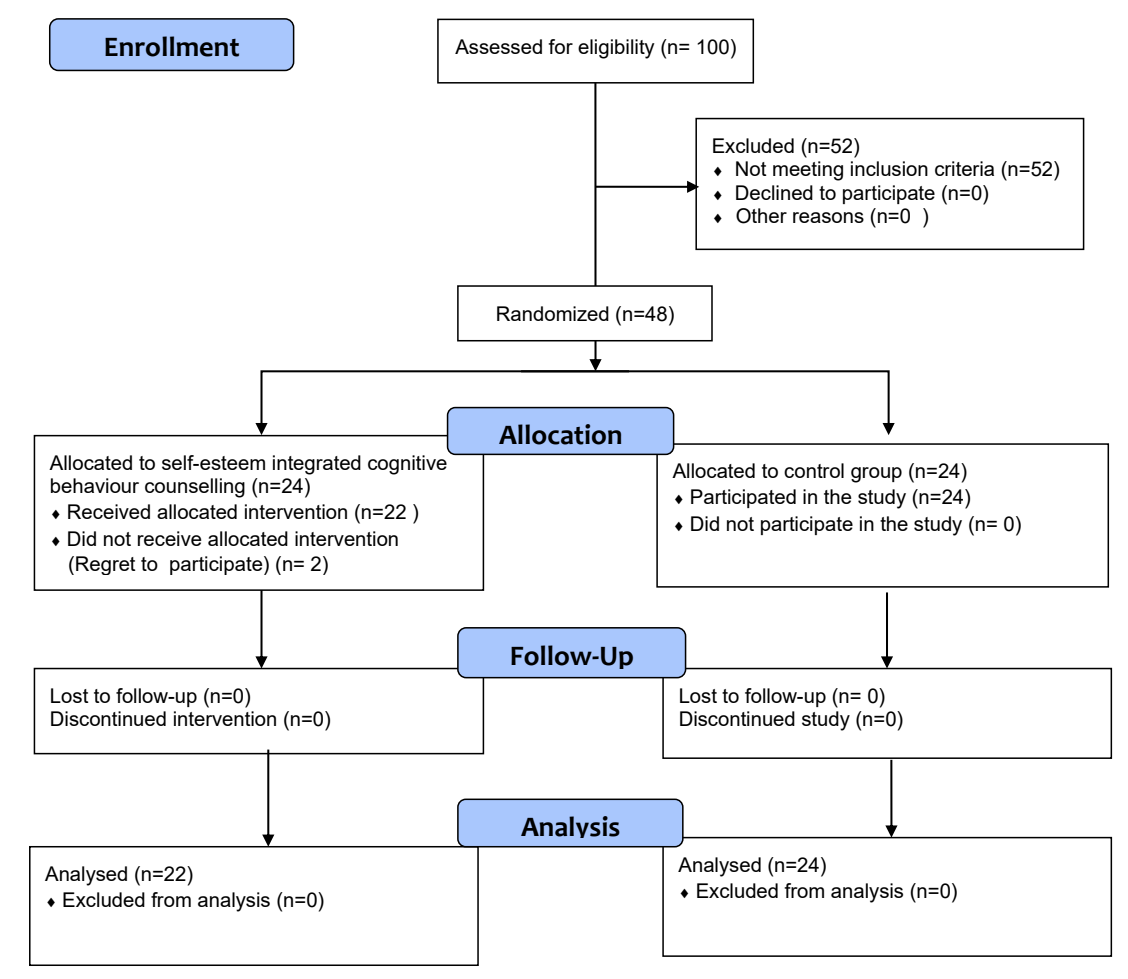

Figure 1. The Study CONSORT Flowchart. 
Table 2. Demographic Characteristics of Pregnant Women in Both Groups

\begin{tabular}{|c|c|c|c|}
\hline Variables & Intervention Group $(\mathrm{n}=\mathbf{2 4})$ & Control Group $(n=24)$ & $P$ Value \\
\hline Age $(y)$, mean $\pm S D$ & $22.83 \pm 0.52$ & $24.084 .08 \pm$ & $0.097^{\mathrm{a}}$ \\
\hline Body mass index $\left(\mathrm{kg} / \mathrm{m}^{2}\right)$, mean $\pm \mathrm{SD}$ & $24.85 \pm 1.13$ & $24.67 \pm 2.42$ & $0.740^{\mathrm{a}}$ \\
\hline Gestational age (wk), mean \pm SD & $22.14 \pm 1.39$ & $22.0 \pm 1.61$ & $0.744^{\mathrm{a}}$ \\
\hline \multicolumn{4}{|l|}{ Education level, No. (\%) } \\
\hline Diploma & $8(33.3)$ & $11(45.8)$ & \multirow{2}{*}{$0.193^{b}$} \\
\hline Academic & $16(66.7)$ & $13(54.2)$ & \\
\hline \multicolumn{4}{|l|}{ Husband's education level, No. (\%) } \\
\hline Diploma & $11(45.8)$ & $7(29.2)$ & \multirow{2}{*}{$0.404^{b}$} \\
\hline Academic & $13(54.2)$ & $17(70.8)$ & \\
\hline \multicolumn{4}{|l|}{ Marital satisfaction, No. (\%) } \\
\hline Satisfied & $14(58.3)$ & $10(41.7)$ & \multirow{2}{*}{$0.238^{b}$} \\
\hline Relatively satisfied & $10(41.7)$ & $14(58.3)$ & \\
\hline \multicolumn{4}{|l|}{ Occupation, No. (\%) } \\
\hline Housework & $17(70.8)$ & $17(70.8)$ & \multirow{2}{*}{$0.610^{b}$} \\
\hline Employed & $7(29.2)$ & $7(29.2)$ & \\
\hline
\end{tabular}

Table 3. Mean of Fear of Childbirth and Self-esteem Between Groups

\begin{tabular}{|c|c|c|c|c|}
\hline Variables & & Intervention Group & Control Group & $P$ Value $^{a}$ \\
\hline \multirow{2}{*}{ Fear of childbirth } & Before intervention & $39.59 \pm 6.68$ & $41.17 \pm 5.07$ & 0.379 \\
\hline & After intervention & $26.45 \pm 4.26$ & $38.58 \pm 5.28$ & 0.001 \\
\hline$P$ value $^{b}$ & \multicolumn{4}{|c|}{0.001} \\
\hline \multirow{2}{*}{ Self-esteem } & Before intervention & $2.18 \pm 2.09$ & $2.31 \pm 1.88$ & 0.678 \\
\hline & After intervention & $4.77 \pm 4.07$ & $2.26 \pm 1.79$ & 0.001 \\
\hline$P$ value ${ }^{\mathrm{b}}$ & \multicolumn{4}{|c|}{0.001} \\
\hline
\end{tabular}

Data presented as mean $\pm \mathrm{SD}$.

${ }^{\mathrm{a}}$ Mann-Whitney test; ${ }^{\mathrm{b}}$ Wilcoxon test.

using relaxation techniques. In a clinical trial investigating the effect of relaxation intervention on the general health of 60 pregnant women in Iran, in addition to routine pregnancy care, four 90-min sessions of progressive muscle relaxation technique courses were conducted during 4 weeks (once a week). The control group only received routine prenatal care. The two groups were analyzed before and after the intervention. The results pointed that a significant increase in the general health in the relaxation group compared to the control group $(P>0.001)(22)$.

In addition, in a study showed that a trained midwife provided psycho-education treatment to reduce FOC in perinatal care. This treatment program (including the support for mothers, management of negative events, the discovery of the source of fear, and having a childbirth program) significantly reduced the childbirth fear, decreased depression, and improved self-efficacy (23). A study showed that cognitive and behavioral techniques significantly reduced the FOC. It is consistent with the present study results, suggesting that combining cognitive therapy with behavioral techniques can reduce childbirth fear (24).

In the present study, two sessions were held on selfesteem and its role in mental health. Studies have shown that self-esteem increases happiness and is a powerful factor in creating satisfaction in life and mental health.
Self-esteem is a valuable feeling that a person has toward himself, and it is the most crucial factor in the mental development of individuals and their mental health (25, 26). One of the characteristics of women who are less afraid of giving birth is having a high level of self-esteem. In contrast, women who were reported to have a high level of childbirth fear believe in the phenomenon of luck instead of accepting their abilities in childbirth; these women were always worried about their body's ability for delivery (27).

In the present study, the birth videotape was used in the last session so that the participants become aware of the processes of labor and natural delivery and respiratory exercises to apply relaxation techniques well in case of pain. Relaxation and positive imagery provide comfort in a natural situation, creating positive feelings for the mothers, leading to endorphins release, feeling sense of euphoria, and reduced FOC (28).

\section{Limitations of the Study}

This study has some limitations that should be mentioned. The generalizing of our results may be limited because we included only nulliparous women with a high school diploma as a minimum level of education. Lack of followup assessment after childbirth due to time constraints is another limitation to this study. 


\section{Conclusions}

The findings showed that cognitive-behavioral group therapy focusing on self-esteem was significantly effective in reducing FOC in the SICB group compared to the control group. This can confirm that the content of this program is appropriate to the main aspects of the psychosocial needs of pregnant nulliparous women in this study. Therefore, it is essential to pay more attention to the cognitive nature of problems resulting in FOC and take into account the strength and power of the group in helping each other modify the cognitive distortions leading to psychological issues such as FOC and low selfesteem. Moreover, considering the high rate of CS delivery, which is a challenging problem in Iran, providing specific counseling for FOC during prenatal care could reduce unnecessary CS deliveries (due to FOC) and improve pregnant women's mental health.

\section{Authors' Contribution}

MP and KV planned the concept and design of this study. MP had full access to all of the data in the study and took responsibility for the data's integrity and accuracy. MP and MK did the acquisition, analysis, and interpretation of data. Abdi $M$ has supervised the session content of the cognitive-behavioral approach. The initial draft of the manuscript was prepared by MK and KV. Final draft was written by MK. Statistical analysis was done by KV and supervised by MK. All authors approved the final manuscript and take responsibility for the integrity of the data.

\section{Conflict of Interests}

Authors declare that they have no conflict of interests.

\section{Ethical Issues}

This paper was extracted from a Master's thesis in midwifery consultation approved by Arak University of Medical Sciences (ethical code: 93176-7). A written informed consent was obtained from the participants before the study, and all the participants' rights were observed according to the Helsinki ethical convention. Also, this project was registered in the Iranian Registry of Clinical Trials database (identifier: IRCT2014120920259N1).

\section{Financial Support}

This study was supported financially by Arak University of Medical Sciences, Arak, Iran.

\section{Acknowledgments}

The authors thank the Research Council of Arak University of Medical Sciences, Arak, Iran, who helped assess the health status of women and promote their health through the approval and funding of this project, also, the staff of Milad hospital, Tehran, Iran for their cooperation in facilitating this research.

\section{References}

1. O'Connell MA, Leahy-Warren P, Kenny LC, O'Neill SM, Khashan AS. The prevalence and risk factors of fear of childbirth among pregnant women: a cross-sectional study in Ireland. Acta Obstet Gynecol Scand. 2019;98(8):1014-1023. doi:10.1111/aogs.13599

2. Vakilian $\mathrm{K}$, Zarin $\mathrm{F}$, Zaraj $\mathrm{H}$. The relationship between perceived social support in pregnancy and self-efficacy for childbirth fear-a cross-sectional study in Arak city, 2017. Open Public Health J. 2018;11(1):546-551. doi:10.2174/1874944501811010546

3. Vakilian $K$, Khorsandi $M$, Jafarimanesh $H$, Ranjbaran $M$. Development and psychometrics of perceived experiences of natural vaginal childbirth in Iranian primiparous women questionnaire. Crescent J Med Biol Sci. 2018;5(2):95-100.

4. Mortazavi F, Agah J. Childbirth fear and associated factors in a sample of pregnant Iranian women. Oman Med J. 2018;33(6):497505. doi:10.5001/omj.2018.91

5. Mousavi SA, Keramat A, Vakilian K, Chaman R. Interpretation of opposite-sex friendship based on social ecology model in Iranian females. Iran J Psychiatry Behav Sci. 2012;6(2):69-78.

6. Phunyammalee M, Buayaem T, Boriboonhirunsarn D. Fear of childbirth and associated factors among low-risk pregnant women. J Obstet Gynaecol. 2019;39(6):763-767. doi:10.1080/01443615. 2019.1584885

7. Mortazavi F, Borzoee F. Fear of childbirth, fatigue, and well-being in pregnant women. J Obstet Gynecol Cancer Res. 2019;4(2):4550. doi:10.30699/jogcr.4.2.45

8. Klabbers GA, Wijma K, Paarlberg KM, Emons WHM, Vingerhoets AJJM. Haptotherapy as a new intervention for treating fear of childbirth: a randomized controlled trial. J Psychosom Obstet Gynaecol. 2019;40(1):38-47. doi:10.1080/016748 2X.2017.1398230

9. Uçar T, Golbasi Z. Effect of an educational program based on cognitive behavioral techniques on fear of childbirth and the birth process. J Psychosom Obstet Gynaecol. 2019;40(2):146-155. doi: 10.1080/0167482x.2018.1453800

10. Çıtak Bilgin N, Coşkun H, Coşkuner Potur D, İbar Aydın E, Uca E. Psychosocial predictors of the fear of childbirth in Turkish pregnant women. J Psychosom Obstet Gynaecol. 2021;42(2):123-131. doi: 10.1080/0167482x.2020.1734791

11. Ghazaei M, Davodi I, Neysi A, Mehrabizadeh Honarmand M, Basak Nejad S. Effectiveness of cognitive behavioral therapy and psycho-education on fear of natural childbirth and its related variables. J Appl Psychol. 2018;23;12(1):103-125.

12. Khorsandi M, Vakilian K, Salehi B, Torabi Goudarzi M, Abdi M. The effects of stress inoculation training on perceived stress in pregnant women. J Health Psychol. 2016;21(12):2977-2982. doi:10.1177/1359105315589800

13. Koyuncu SB, Bülbül M. The impact of yoga on fear of childbirth and childbirth self-efficacy among third trimester pregnants. Complement Ther Clin Pract. 2021;44:101438. doi:10.1016/j. ctcp.2021.101438

14. Vakilian K, Ranjbaran M, Khorsandi M, Sharafkhani N, Khodadost M. Prevalence of preterm labor in Iran: a systematic review and meta-analysis. Int J Reprod Biomed. 2015;13(12):743-748. doi:10.29252/ijrm.13.12.743

15. Saisto T, Salmela-Aro K, Nurmi JE, Könönen T, Halmesmäki E. A randomized controlled trial of intervention in fear of childbirth. Obstet Gynecol. 2001;98(5 Pt 1):820-826. doi:10.1016/s00297844(01)01552-6

16. Ledley DR, Marx BP, Heimberg RG. Making Cognitive-Behavioral Therapy Work: Clinical Process for New Practitioners. 2nd ed. New York: Guilford Press; 2010.

17. Vakilian K, Kheiri M, Majidi A. Effect of cognitive-behavioral sexual counseling on female sexual function during pregnancy: an interventional study. Int J Womens Health Reprod Sci. 2018;6(3):369-373. doi:10.15296/ijwhr.2018.60

18. Khorsandi M, Ghofranipour F, Heydarnia A, et al. The effect of childbirth preparation classes on childbirth fear and normal delivery among primiparous women. J Arak Univ Med Sci. 2008;11(3):29-36. [Persian].

19. Khorsandi M, Vakilian K, Torabi Goudarzi M, Abdi M. Childbirth preparation using behavioral-cognitive skill in childbirth outcomes of primiparous women. J Babol Univ Med Sci. 2013;15(4):76-80. [Persian].

20. Naseri L, Mohamadi J, Sayehmiri K, Azizpoor Y. Perceived social support, self-esteem, and internet addiction among students of Al-Zahra University, Tehran, Iran. Iran J Psychiatry Behav Sci. 2015;9(3):e421. doi:10.17795/ijpbs-421

21. Rouhe H, Salmela-Aro K, Toivanen R, et al. Group psychoeducation with relaxation for severe fear of childbirth improves maternal adjustment and childbirth experience--a randomised controlled trial. J Psychosom Obstet Gynaecol. 2015;36(1):1-9. doi:10.310 9/0167482x.2014.980722 
22. Sadeghi A, Sirati-Nir M, Ebadi A, Aliasgari M, Hajiamini Z. The effect of progressive muscle relaxation on pregnant women's general health. Iran J Nurs Midwifery Res. 2015;20(6):655-660. doi:10.4103/1735-9066.170005

23. Toohill J, Fenwick J, Gamble J, et al. A randomized controlled trial of a psycho-education intervention by midwives in reducing childbirth fear in pregnant women. Birth. 2014;41(4):384-394. doi:10.1111/birt.12136

24. Sydsjö G, Sydsjö A, Gunnervik C, Bladh M, Josefsson A. Obstetric outcome for women who received individualized treatment for fear of childbirth during pregnancy. Acta Obstet Gynecol Scand. 2012;91(1):44-49. doi:10.1111/j.1600-0412.2011.01242.x

25. Marshall WL, Marshall LE, Serran GA, O'Brien MD. Self-esteem, shame, cognitive distortions and empathy in sexual offenders: their integration and treatment implications. Psychol Crime Law. 2009;15(2-3):217-234. doi:10.1080/10683160802190947

26. Serretti A, Olgiati P, Colombo C. Components of self-esteem in affective patients and non-psychiatric controls. J Affect Disord. 2005;88(1):93-98. doi:10.1016/j.jad.2005.05.008

27. Fenwick J, Gamble J, Creedy DK, et al. Study protocol for reducing childbirth fear: a midwife-led psycho-education intervention. BMC Pregnancy Childbirth. 2013;13:190. doi:10.1186/14712393-13-190

28. Nasiri S, Akbari H, Tagharrobi L, Tabatabaee AS. The effect of progressive muscle relaxation and guided imagery on stress, anxiety, and depression of pregnant women referred to health centers. J Educ Health Promot. 2018;7:41. doi:10.4103/jehp. jehp_158_16

(C) 2022 The Author(s); This is an open-access article distributed under the terms of the Creative Commons Attribution License (http:// creativecommons.org/licenses/by/4.0), which permits unrestricted use, distribution, and reproduction in any medium, provided the original work is properly cited. 